\title{
662 DISSECTING THE CD226 IMMUNE AXIS IN THE TUMOR MICROENVIRONMENT USING CYTOF-BASED HIGH- DIMENSIONAL IMMUNOPHENOTYPING
}

Katie Vowell ${ }^{*}$, Michael Conner, Florence Perrin, Paul Bojzzuk, Kenneth Hance, Iris Roth, Christine Donahue, James Smothers, Jeremy Waight. GlaxoSmithKline, Collegeville, PA, United States

Background In recent years, a regulatory network involving nectin/nectin-like immune receptors has emerged as a potential point of manipulation for cancer immunotherapy. Central to this axis, CD226 (DNAM-1) is a T and NK cell co-stimulatory receptor that competes for ligand (CD155 and CD112) binding with multiple inhibitory receptors (TIGIT, CD96, and PVRIG [CD112R]). Despite a large body of literature for TIGIT, detailed cellular characterization of the entire axis is still lacking. Therefore, we used mass cytometry (CyTOF) to systematically evaluate expression of the CD226 axis in tumors from a range of indications.

Methods To thoroughly characterize the CD226 axis in the tumor microenvironment, we immunophenotyped approximately 100 tumor samples derived from a variety of cancer types using a bespoke 46-parameter CyTOF panel. Human biological samples were sourced ethically and their research use was in accord with the terms of the informed consents under an IRB/EC approved protocol. Using a suite of highdimensional analytical tools, including FlowSOM, UMAP, and tSNE, we revealed distinct expression profiles for each receptor; a finding that was previously obscured due to a lack of sufficient resolution.

Results We observed a notable divergence in expression profiles between the CD226 axis members across tumor indications. For example, TIGIT expression was found to be highest on activated $\mathrm{CD} 4+$ regulatory $\mathrm{T}$ (Treg) cells, where its expression correlated strongly with ICOS, FoxP3, CD25, and CCR8. By contrast, CD96 and PVRIG exhibited broad expression across intratumoral $\mathrm{T}$ and NK cell populations. Other receptors (e.g., CD226) demonstrated variegated expression profiles across $\mathrm{T}$ and NK cell subsets. Finally, despite relatively consistent expression profiles of certain CD226 axis (i.e., TIGIT on Treg cells) across tumors, we also found several cell subsets/ clusters unique to specific indications.

Conclusions Using high-parameter CyTOF analysis, we were able to thoroughly characterize the CD226 axis (CD226, TIGIT, CD96, PVRIG) and related immune receptors across a range of tumor indications. These analyses revealed divergent expression profiles for each CD226 axis member, suggesting distinct/contextual biological role(s) for each receptor. However, future studies will need to dissect the importance of the distinct cellular representation for each CD226 axis member.

Ethics Approval All samples were purchased from Discovery Life Sciences (DLS). DLS represents and warrants that it has ownership of all Products available for sale and has properly obtained, where required under HHS/OHRP 45 CFR 46.102 (d) (f), IRB approval (or appropriate research approval for institutions outside the U.S.) for study protocols and informed consent documents for all human subject derived biological materials.

http://dx.doi.org/10.1136/jitc-2021-SITC2021.662 